\title{
Production of specific monoclonal antibody against CD3 $\varepsilon$ in olive flounder
}

\author{
Jae Wook Jung*, Young Rim Kim*, Jung Seok Lee*, Se Pyeong Lim*, Si Won Kim*, Jassy Mary S. Lazarte*, Jaesung Kim*, \\ Tae Sung Jung $\$$ \\ Laboratory of Aquatic Animal Diseases, College of Veterinary Medicine, Gyeongsang National University \\ 900 Gajwadong, Jinju, Gyeongnam, South Korea, 660-701
}

\begin{abstract}
T cell activation is initiated by the binding of antigen to the specific $\mathrm{T}$ cell receptor (TCR) that triggers the formation of CD3-signal transduction complex and ends in proliferation and differentiation of antigenspecific T cells. CD3, an essential component of the CD3-TCR complex, has been classified into $\gamma, \delta, \varepsilon$, and $\zeta$ chains. The $\mathrm{CD} 3 \gamma, \mathrm{CD} 3 \delta$, and $\mathrm{CD} 3 \varepsilon$ chains are highly related to cell surface proteins of the immunoglobulin superfamily containing a single extracellular immunoglobulin domain. In mammals, $\mathrm{T}$ cells were characterized by detecting one of the CD3 molecules through the use of specific antibody for CD3e. This suggests that CD3 molecules can be used as markers in identifying T cells in teleost fish; hence, it is essential to produce CD3-specific antibodies that could serve as $\mathrm{T}$ cell markers in fish. Here, we produced a CD3E-specific monoclonal antibody. Western blot result showed a distinct band at approximately $15 \mathrm{kDa}$ detecting the $\mathrm{CD} 3 \varepsilon$ antibody while MALDI-TOF analysis, clearly identified the band as olive flounder CD3e. Once a monoclonal antibody has been produced, it can be used to detect the presence of a substance specific to it. The results from this study revealed that the CD3e monoclonal antibody produced could detect CD3 $\varepsilon$ in olive flounder. However, cell markers for T cell differentiation in olive flounder were not fully investigated yet so we hope that this antibody could be useful in elucidating $\mathrm{T}$ cell differentiation in teleosts.
\end{abstract}

\section{KEYWORDS}

T cell activation, $\mathrm{CD} 3 \varepsilon$, teleost fish, monoclonal antibody, cell marker

* These authors have contributed equally to this work.

$\S$ Corresponding author. Tel.: +82-55-772-2350; Fax: +82-55-762-6733.

E-mail address: jungts@gnu.ac.kr/jungts@gmail.com 